05,12

\title{
Мультистабильность дипольной наноячейки при ее импульсном перемагничивании
}

\author{
(C) А.М. Шутый, Д.И. Семенцов \\ Ульяновский государственный университет, \\ Ульяновск, Россия \\ E-mail: shuty@mail.ru \\ Поступила в Редакцию 4 мая 2020 г. \\ В окончательной редакции 4 мая 2020 г. \\ Принята к публикации 11 мая 2020 г.
}

Исследована динамика перемагничивания наноячейки, составленной из двух магнитоодноосных наночастиц, коротким гауссовым импульсом магнитного поля. Такая дипольно связанная система мультистабильна и имеет четыре равновесных состояния. Выявлены условия, при которых наноячейка перемагничивается в одно из трех состояний или возвращается к исходной конфигурации. Показано, что характер прецессионной динамики магнитного момента и выбор конечного состояния наноячейки зависят от амплитуды и длительности импульса, а также от взаимной ориентации легких осей.

Ключевые слова: импульсное перемагничивание; прецессионная динамика; одноосная анизотропия; магнитная наночастица; мультистабильные состояния.

DOI: 10.21883/FTT.2020.10.49914.104

\section{1. Введение}

Дискретность решеточных структур наночастиц, связанных преимущественно диполь-дипольным взаимодействием, приводит к существенным отличиям их равновесных состояний и динамического поведения от аналогичных свойств макроскопических монодоменных объектов [1-5]. На изменении равновесной конфигурации магнитных моментов под воздействием импульса магнитного поля основана возможность записи информации на решетках магнитных диполей [6,7]. Импульсному перемагничиванию магнитных микро- и нано(по одному из размеров) систем посвящено значительное число как теоретических, так и экспериментальных работ. Так, в [8] экспериментально была обнаружена периодичность реализации перемагничивания планарной слоистой микроструктуры при изменении длительности и амплитуды импульса. В [9] экспериментально исследуется продолжительность прецессионного отклика намагниченности микроячейки памяти на импульсное воздействие. В работах [10-13] исследована динамика намагниченности антиферромагнитных систем под влиянием сверхбыстрых импульсов магнитного поля. В частности, в [10] продемонстрирована возможность перемагничивания системы с длительным, а в [13] - с коротким релаксационным процессом. В [14] на основе уравнения Ландау-Лифшица рассмотрено переключение намагниченности монодоменных наночастиц и предложена схема для ее приведения в заданное состояние с помощью коротких импульсов магнитного поля. В работах [15-17] исследу- ется импульсное перемагничивание пленок с различным типом магнитной анизотропии, а также рассматриваются динамические петли гистерезиса и релаксационные эффекты при импульсном перемагничивании наночастиц.

Две ферромагнитные нанопленки с антипараллельной связью, обусловленной межслойным магнитным обменом или диполь-дипольным взаимодействием, представляют собой наноячейку синтетического антиферромагнетика и лежат в основе логических элементов магнитной оперативной памяти. Отличительной чертой таких систем является высокая стабильность магнитного состояния к тепловым флуктуациям при высокой компенсации магнитных полей рассеивания $[13,18]$. В работе [19] исследуется отклик на гауссов импульс магнитного поля системы двух наночастиц, обладающих различной по величине одноосной магнитной анизотропией.

В настоящей работе на основе численного решения динамических уравнений исследуется импульсное перемагничивание системы двух дипольно-связанных магнитоодноосных наночастиц с одинаковыми или отличающимися величинами магнитного момента и константы анизотропии, имеющими в исходном состоянии параллельную или ортогональную ориентацию легких осей. Учитывая периодическую зависимость реализации перемагничивания наночастиц от параметров импульса [19-21], исследуется прецессионная динамика отклика магнитных моментов на импульсное воздействие и выявляются условия различных режимов перемагничивания наноячейки для различных исходных конфигураций магнитных моментов. 


\section{2. Исходные уравнения}

Рассмотрим наноячейку, состоящую из двух наночастиц с магнитными моментами $\mathbf{m}_{i}=m_{i}($ где $i=1,2)$. Считаем, что наночастицы связаны диполь-дипольным взаимодействием и их центры расположены друг от друга на расстоянии $r$. Каждая из наночастиц обладает одноосной магнитной анизотропией и имеет размер, при котором находится в однодоменном состоянии. Энергию $i$-ой наночастицы запишем в виде суммы энергий во внешнем магнитном поле $\mathbf{H}$, диполь-дипольного взаимодействия, анизотропии и полей рассеяния

$$
W\left(\mathbf{m}_{i}\right)=-\mathbf{m}_{i} \mathbf{H}+W_{d}\left(\mathbf{m}_{1,2}\right)+W_{a}\left(\mathbf{m}_{i}\right)+W_{s}\left(\mathbf{m}_{i}\right) .
$$

Здесь энергия диполь-дипольного взаимодействия

$$
W_{d}\left(\mathbf{m}_{1,2}\right)=\frac{\mathbf{m}_{1} \mathbf{m}_{2} r^{2}-3\left(\mathbf{m}_{1} \mathbf{r}\right)\left(\mathbf{m}_{2} \mathbf{r}\right)}{r^{5}},
$$

где $\mathbf{r}$ - радиус-вектор между диполями под номерами 1 и 2; энергия одноосной анизотропии

$$
W_{a}\left(\mathbf{m}_{i}\right)=-K_{i u} V \frac{\left(\mathbf{m}_{i} \mathbf{n}\right)^{2}}{m_{i}^{2}},
$$

где $K_{i и}$ и $\mathbf{n}-$ константа одноосной анизотропии и орт оси легкого намагничивания, $V$ - объем каждой наночастицы; энергия полей рассеяния

$$
W_{s}\left(\mathbf{m}_{i}\right)=\frac{\mathbf{m}_{i} \hat{N} \mathbf{m}_{i}}{2 V},
$$

где $\hat{N}$ - тензор размагничивающих коэффициентов. Далее форму частиц считаем сферической, поэтому компоненты $N_{x}=N_{y}=N_{z}=4 \pi / 3$.

Динамика каждого из магнитных моментов описывается уравнением Ландау-Лифшица с релаксационным членом в форме Гильберта [23]:

$$
\frac{\partial \mathbf{m}_{i}}{\partial t}=-\gamma \mathbf{m}_{i} \times \mathbf{H}_{i}^{\mathrm{ef}}+\frac{\alpha}{m_{i}} \mathbf{m}_{i} \times \frac{\partial \mathbf{m}_{i}}{\partial t},
$$

где $\gamma$ - гиромагнитное отношение, $\alpha$ - параметр диссипации. Эффективное магнитное поле, создаваемое в месте расположения $i$-го диполя $n$-ым диполем, внешним полем и полем анизотропии, имеет вид

$$
\begin{aligned}
\mathbf{H}_{i}^{\mathrm{ef}} & =-\frac{\partial W_{i}}{\partial \mathbf{m}_{i}} \\
& =\mathbf{H}+K_{i u} V \frac{\mathbf{n}\left(\mathbf{m}_{i} \mathbf{n}\right)}{m_{i}^{2}}+\frac{3\left(\mathbf{m}_{n} \mathbf{r}\right) \mathbf{r}-\mathbf{m}_{n} r^{2}}{r^{5}}-\frac{4 \pi}{3} \frac{\mathbf{m}_{i}}{V} .
\end{aligned}
$$

Далее перейдем к безразмерным параметрам: $\boldsymbol{\mu}_{i}=\mathbf{m}_{i} / m$. $\mathbf{e}=\mathbf{r} / r, \tau=\gamma J t, r_{0}=r /(V)^{1 / 3}$, где $J=m / V$ - намагниченность наночастицы. В безразмерных параметрах уравнения (4) принимают вид

$$
\frac{\partial \boldsymbol{\mu}_{i}}{\partial \tau}=-\boldsymbol{\mu}_{i} \times \mathbf{h}_{i}^{\mathrm{ef}}+\alpha \boldsymbol{\mu}_{i} \times \frac{\partial \boldsymbol{\mu}_{i}}{\partial \tau},
$$

где

$$
\mathbf{h}_{i}^{\mathrm{ef}}=\mathbf{h}+k_{h i} \mathbf{n}\left(\boldsymbol{\mu}_{i} \mathbf{n}\right)+\frac{3\left(\boldsymbol{\mu}_{n} \mathbf{e}\right) \mathbf{e}-\boldsymbol{\mu}_{n}}{r_{0}^{3}}-\boldsymbol{\mu}_{i} .
$$

В этом случае обезразмеренные внешнее поле и константа одноосной анизотропии принимают вид: $\mathbf{h}=\mathbf{H} / j$, $k_{i u}=K_{i u} / J^{2}$.

При дальнейшем анализе векторное уравнение (7) может быть представлено тремя скалярными уравнениями. Так, для $x$-компонент $\partial \boldsymbol{\mu}_{i} / \partial \tau$ получаем

$$
\begin{gathered}
\left(1+\alpha^{2}\right) \frac{\partial \mu_{i x}}{\partial \tau}=\left(\mu_{i z}-\alpha \mu_{i x} \mu_{i y}\right) h_{i y}^{\mathrm{ef}}-\left(\mu_{i y}+\alpha \mu_{i z} \mu_{i x}\right) h_{i z}^{\mathrm{ef}} \\
+\alpha\left(1-\mu_{i x}^{2}\right) h_{i x}^{\mathrm{ef}}
\end{gathered}
$$

Уравнения для остальных компонент имеют аналогичный вид и могут быть получены циклической перестановкой составляющих.

Далее будем считать, что центры двух наночастиц в ячейке лежат на оси $Z$. Рассмотрим два случая: наночастицы отличаются величиной магнитного момента, а их константы анизотропии одинаковы $\left(k_{u}=1\right)$, направления легких осей совпадают с осью $Y$, (или с осью $Z$ ); наночастицы идентичны, но их легкие оси перпендикулярны друг другу (ось первой частицы ориентирована по оси $Y$, а второй - по оси $Z$ ).

Безразмерный параметр диссипации принимаем равным $\alpha=0.01$. Равновесные ориентации и прецессионные режимы суммарного магнитного момента наноячейки определяются на основе численного решения уравнений (7), который проводится с помощью метода Рунге-Кутта.

\section{3. Равновесные ориентации наночастиц}

Рассмотрим ориентацию суммарного магнитного момента $\mathbf{M}=\boldsymbol{\mu}_{1}+\boldsymbol{\mu}_{2}$ наноячейки в зависимости от расстояния между наночастицами. На рис. 1 приведены зависимости $y$-компоненты вектора М от параметра $r_{0}$ и конфигурации наноячейки при $r_{0}=1,4$ для четырех случаев: легкие оси наночастиц сонаправлены с осью $Y$, $\mu_{i}=1$ (1) и наночастицы с $\mu_{1}=1.5, \mu_{2}=0.5$ (2); легкие оси перпендикулярны друг к другу, $\mu_{i}=1$ (3) и $\mu_{1}=1.5$, $\mu_{2}=0.5$ (4). Из приведенных зависимостей следует, что в случае сонаправленных осей анизотропии (случаи 1,2 ) магнитные моменты остаются сонаправленными при сближении наночастиц до значений $r_{0} \approx 1.5$. При дальнейшем их сближении реализуется резкое изменение направления магнитного момента одной из наночастиц на противоположное (из двух различных наночастиц меняет ориентацию наночастица с меньшим магнитным моментом). В случае перпендикулярных осей анизотропии сближение различных по намагниченности наночастиц приводит к относительно плавному уменьшению $y$-компоненты суммарного магнитного момента (4), т.е. 
магнитные моменты наночастиц оказываются направленными под углом к осям легкого намагничивания. При двух одинаковых наночастицах их сближение приводит вначале к медленному, а затем к быстрому уменьшению до нуля $y$-компоненты суммарного магнитного момента - оба магнитных момента выстраиванию вдоль оси системы, т.е. вдоль оси $Z$ (3). Из рисунка также видно, что при $r_{0} \geq 4$ ориентация магнитных моментов во всех случаях близка к ориентации изолированных наночастиц, так как диполь-дипольное взаимодействие становится достаточно слабым, что и определяет расстояние между слабо взаимодействующими наноячейками решетки.

На рис. 2 приведены петли гистерезиса для наноячейки из двух наночастиц с осями анизотропии, направленными по оси $Y(a)$, и для случая, когда ось анизотропии первой частицы направлена вдоль оси $Y$, а ось второй наночастицы - вдоль оси $Z(b)$, при этом расстояние между наночастицами $r_{0}=2$, а их магнитные моменты $\mu_{i}=1$ (петли 1) и $\mu_{1}=1.5, \mu_{2}=0.5$ (петли 2). Внешнее поле принимается направленным вдоль оси $Y$. Видно, что в случае наночастиц с отличающимися магнитными моментами петли гистерезиса состоят из двух ступенек: при однонаправленном изменении поля имеют место два стационарных участка и два участка резкого изменения намагниченности наноячейки. В случае одинаковых наночастиц петли гистерезиса прямоугольны при сонаправленных осях анизотропии, если оси перпендикулярны, половина петли содержит один стационарный уча-

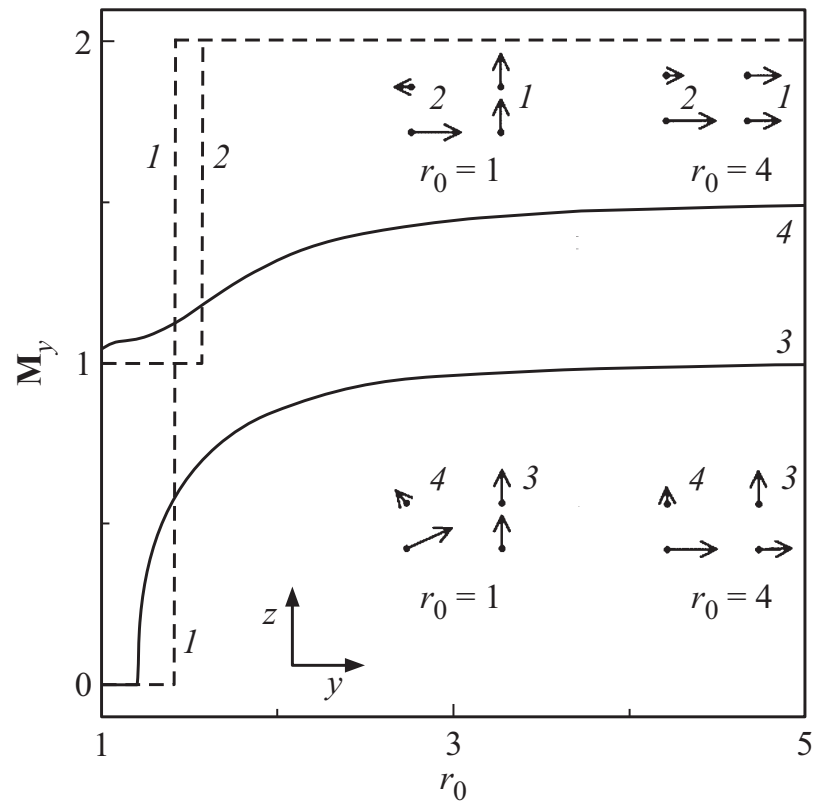

Рис. 1. Зависимости $y$-компоненты суммарного магнитного момента наноячейки от расстояния между наночастицами и конфигурации наноячейки при $r_{0}=1,4$ для наночастиц с сонаправленными осями анизотропии вдоль оси $Y$ при $\mu_{i}=1(1)$ и $\mu_{1}=1.5, \mu_{2}=0.5(2)$ и для случая перпендикулярных осей анизотропии при $\mu_{i}=1$ (3) и $\mu_{1}=1.5, \mu_{2}=0.5$ (4).
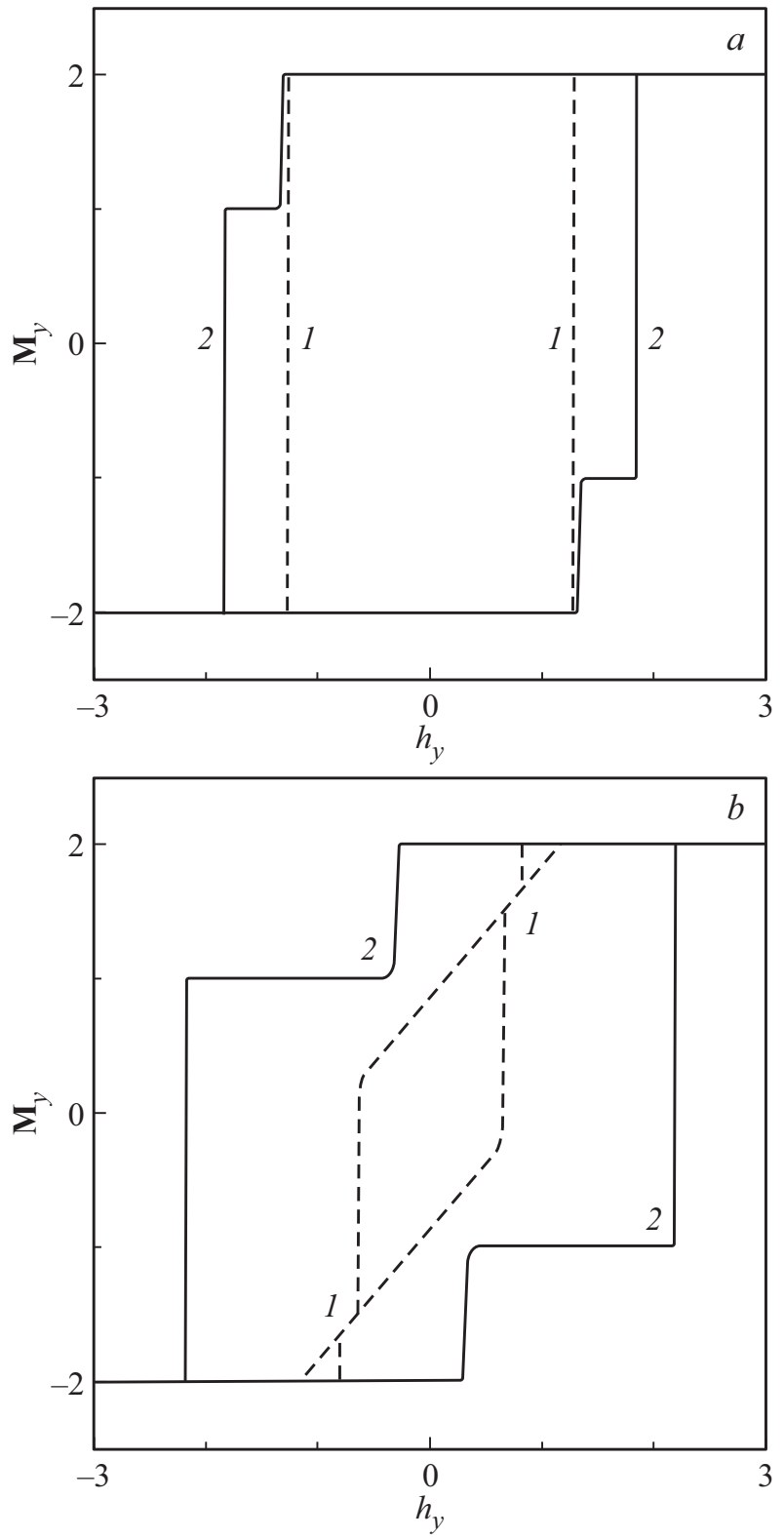

Рис. 2. Петли гистерезиса наноячейки с легкими осями наночастиц, направленными по оси $Y(a)$, с перпендикулярными легкими осями $(b)$, при $r_{0}=2$ и $\mu_{i}=1$ (петли 1 ) и $\mu_{1}=1.5$, $\mu_{2}=0.5$ (петли 2).

сток и по два участка быстрого и линейного изменении магнитного момента наноячейки.

\section{4. Отклик магнитных моментов на импульс поля}

Исследуем отклик магнитного момента наноячейки на гауссов импульс магнитного поля

$$
h(\tau)=h_{0} \exp \left\lfloor-\left(\tau-\tau_{m}\right)^{2} / \tau_{0}^{2}\right\rfloor,
$$

где $h_{0}, \tau_{m}$ и $\tau_{0}$ - пиковое значение поля, временной сдвиг максимума импульса и его длительность; далее 

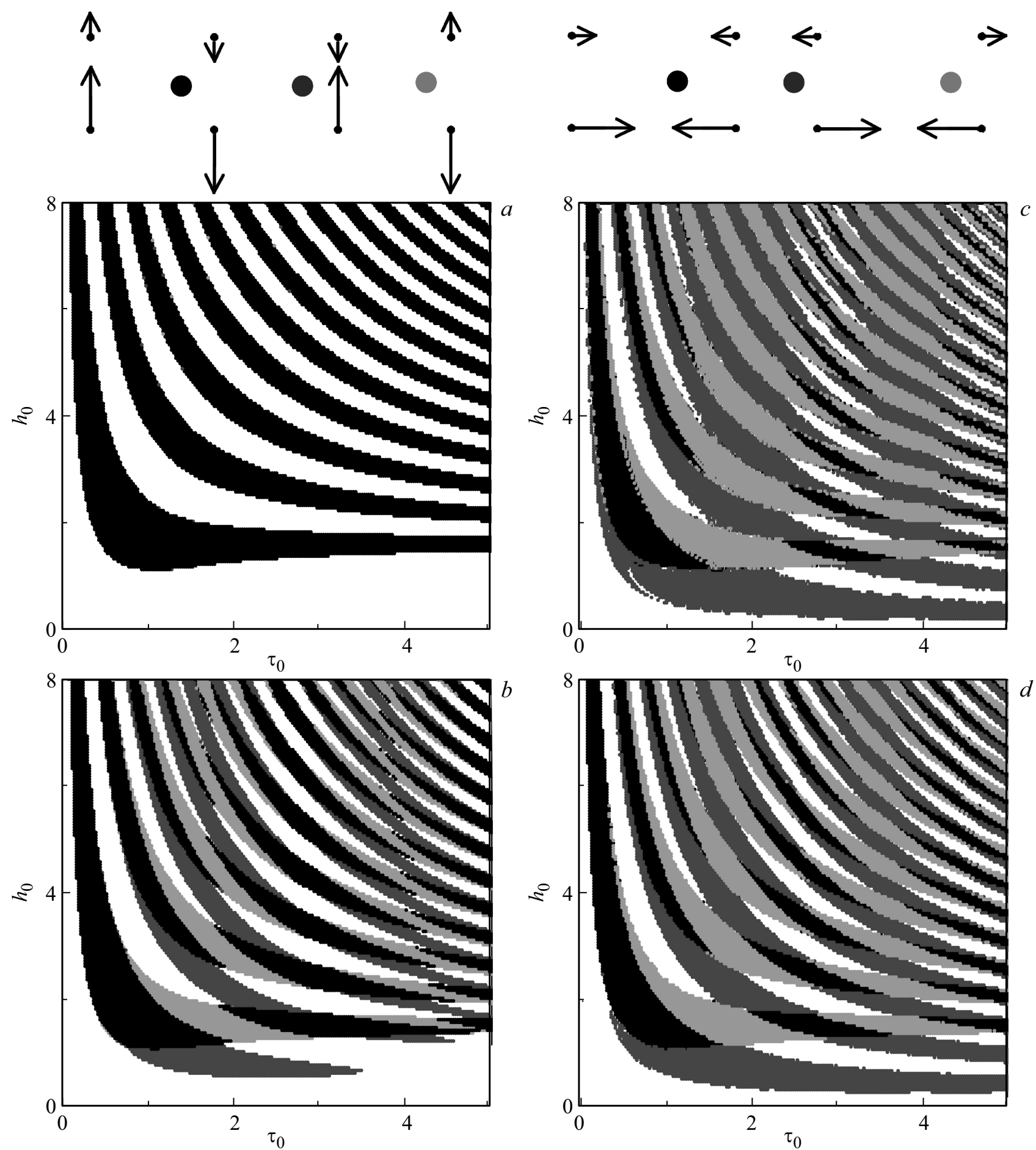

Рис. 3. Диаграммы зависимости перемагничивания наноячейки с $\mu_{1}=1.5, \mu_{2}=0.5$ от амплитуды и длительности гауссова импульса; оси анизотропии наночастиц ориентированы вдоль оси $Z(a, b)$ и оси $Y(c, d)$, параметр $r_{0}=2(a, c), r_{0}=3(b, d)$; вверху диаграмм приведены начальная (слева) и конечные конфигурации наноячейки и отвечающие им цвета областей диаграмм.

принимается $\tau_{m}=200$, а поле импульса линейно поляризованным вдоль оси $X$. Известно, что в случае отсутствия подмагничивающего поля или при относительно слабом поле $\left(h_{y} \ll k_{u}\right)$, направленном вдоль оси легкого намагничивания, с изменением длительности или пикового значения импульса периодически выполняются условия реализации перемагничивания одиночной наночастицы [20-22]. Вначале рассмотрим отклик наноячейки, состоящей из двух различных по намагниченности наночастиц с сонаправленными осями анизотропии.

На рис. 3 приведены диаграммы, отражающие зависимость реализации перемагничивания наноячейки с 
магнитными моментами наночастиц $\mu_{1}=1.5, \mu_{2}=0.5$ от амплитуды и длительности гауссова импульса. Оси анизотропии обеих наночастиц ориентированы вдоль оси $Z(a, b)$ и вдоль оси $Y(c, d)$, а расстояние между наночастицами $r_{0}=2(a, c), r_{0}=3(b, d)$. На диаграммах белые области отвечают сохранение исходной ориентации наночастиц (левые конфигурации, изображенные вверху диаграмм), черные области соответствуют осуществлению перемагничивания обеих наночастиц наноячейки при данных параметрах импульса, а два вида серых областей - перемагничиванию либо только первой, либо только второй наночастицы. Вверху диаграмм приведены конечные конфигурации дипольной наноячейки и отвечающие им цвета областей диаграмм $(a, b) /(c, d)$. Подмагничивающее поле здесь и далее принимается отсутствующим. Видно, что в случае ориентации осей анизотропия вдоль оси наноячейки, т.е. оси $Z$, при близком расположении наночастиц сильное диполь-дипольное взаимодействие приводит к перемагничиванию только всей наноячейки. Однако уже при $r_{0}=3$ дипольное взаимодействие оказывается настолько слабым, что отклик наночастиц на действие импульса близок к отклику изолированных (отличающихся по величине магнитного момента) наночастиц, в результате чего возникают области параметров импульса, отвечающие перемагничиванию только одной из входящих в наноячейку наночастиц. В случае ориентации осей анизотропии перпендикулярно оси наноячейки (вдоль оси $Y$ ) при сильном дипольдипольном взаимодействии однонаправленная ориентация магнитных моментов становится неустойчивой и выстраивается конфигурация с противоположно ориентированными диполями. При $r_{0}=2$ исходная однонаправленная конфигурация еще сохраняет устойчивость, но действие импульса приводит к преимущественному перемагничиванию либо только первой, либо только второй наночастицы. И лишь в малых областях параметров импульса условия соответствует перемагничиванию (или неперемагничиванию) обеих наночастиц. При увеличении параметра $r_{0}$ и ослаблении дипольного взаимодействия области, отвечающие перемагничиванию всей наноячейки, возрастают. Вместе с этим влияние диполь-дипольного взаимодействия приводит к тому, что при $r_{0}=3$ перемагничивание только одной из наночастиц осуществляется в большем диапазоне параметров импульса, чем в такой же наноячейке с осями анизотропии, ориентированными вдоль оси $Z$.

На рис. 4 приведены проекции траектории магнитного момента наноячейки, содержащей вышерассмотренные наночастицы с осью анизотропии вдоль оси $Y$, при действии импульса с амплитудой $h_{0}=2$ и различной продолжительностью $\tau_{0}=0.7,1.2,2.0,3.0$ (кривые $1-4$ ). Случай 1 отвечает перемагничиванию всей наноячейки, в случаях 2 и 3 перемагничиваются только первая и только вторая наночастицы, соответственно, в случае 4 конфигурация наноячейки сохраняется.

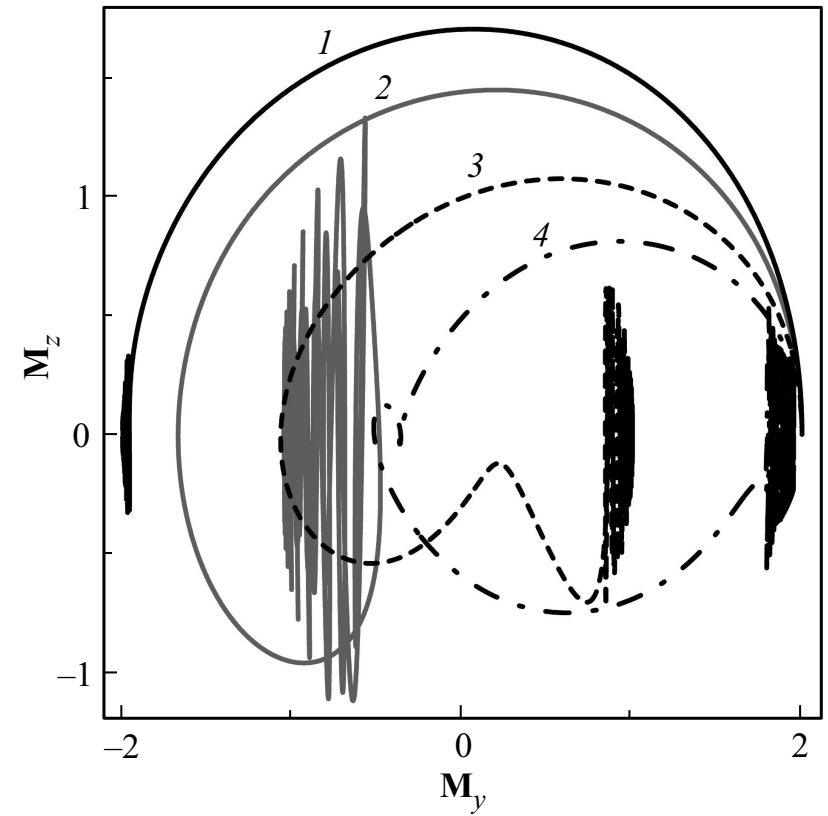

Рис. 4. Проекции траектории магнитного момента наноячейки с $\mu_{1,2}=1.5,0.5$ и осью анизотропии вдоль оси $Y$ при действии импульса с $h_{0}=2$ и $\tau_{0}=0.7,1.2,2.0,3.0$ (кривые $1-4$ ).

Для более подробного рассмотрения прецессионной динамики магнитных моментов при действии импульса поля на рис. 5 приведены проекции траекторий суммарного магнитного момента наноячейки с осями анизотропии частиц вдоль оси $Z$ при (случай $b$ на рис. 3 ) и действии импульса с $\tau_{0}=5, h_{0}=1.5,1.7,36(a-c)$. Случаи $(a)$ и $(c)$ отвечают полному перемагничиванию наноячейки, в случае $(b)$ перемагничивается только вторая наночастица (несмотря на достаточно близкие параметры импульса в двух первых случаях) - на рисунке даны начальная и конечные ориентации магнитных моментов наночастиц. Во всех приведенных случаях реализуется продолжительный прецессионный отклик магнитных моментов, что объясняется близостью параметров импульса к краю полос диаграммы перемагничивания [21]. Короткий прецессионный отклик (см., например, кривую 1 на рис. 4) имеет место, если параметры импульса отвечают центральным областям полос диаграммы. Для начальной динамики отклика суммарного магнитного момента наночастиц, связанных диполь-дипольным взаимодействием, характерным является также затухающая прецессия с центром, смещающимся по спирали, что в большей степени проявляется в случаях $(b)$ и $(c)$.

Из рисунка также видно, что в общем случае возникает два переходных прецессионных процесса: помимо указанной прецессии, возникающей после действия импульса под воздействием поля анизотропии, имеет место также непродолжительная прецессия вокруг оси $\mathrm{X}$ под воздействием поля импульса. Число витков последней возрастает с увеличением параметров импульса поля, 

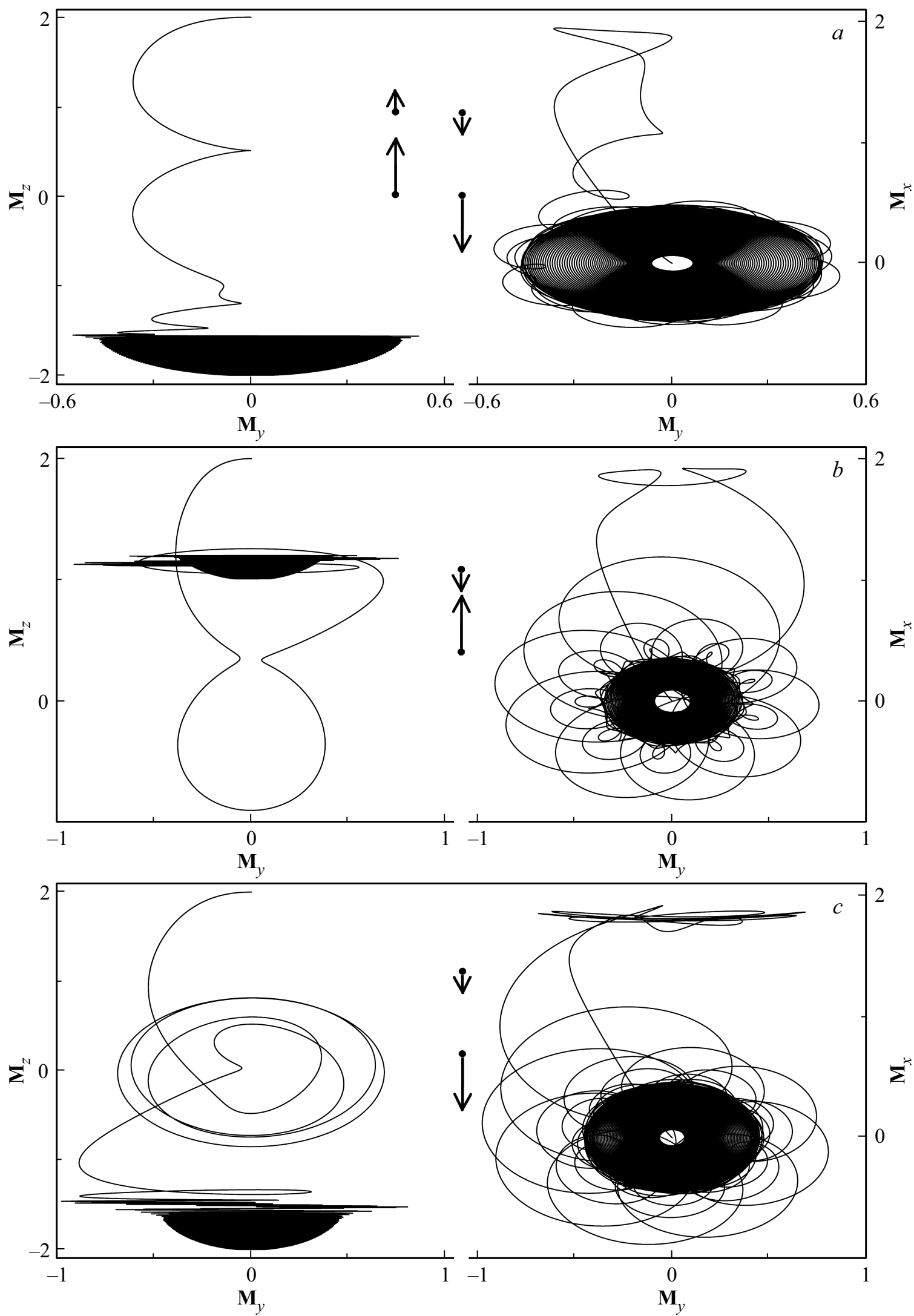

Pис. 5. Проекции траекторий суммарного магнитного момента наноячейки с осями анизотропии частиц вдоль оси $Z$ при действии импульса с $\tau_{0}=5, h_{0}=1.5,1.7,36(a-c) ; r_{0}=3$. 
точнее, с увеличение номера полосы диаграммы перемагничивания. Например, при параметрах, отвечающих первой по счету полосе диаграммы - случаи $(a)$ и (b) - прецессия почти не проявляется. Случай же $(c)$ отвечает краю пятой полосы диаграммы, поэтому реализуется четыре полных оборота магнитного момента вокруг оси $X$.

На рис. 6 приведены зависимости от времени модуля суммарного магнитного момента наноячейки (кривая 1) и его $y$-, z-компонент (кривые 2 и 3) для параметров, отвечающих случаю (c) рис. 5. Из рисунка видно, что прецессионная динамика затрагивает, главным образом, $y$-компоненту магнитного момента (а также $x$-компоненту, не представленную на рисунке). При этом $z$-компонента монотонно изменяется, меняя знак на противоположный. В течении действия импульса данное изменение быстрое, а затем $z$-компонента магнитного момента медленное приближается к равновесному значению. Модуль магнитного момента только вначале отклика проявляет малую по амплитуде прецессионную динамику.

Далее рассмотрим наноячейку, состоящую из двух одинаковых наночастиц (с $\left.\mu_{1,2}=1\right)$, оси анизотропии которых перпендикулярны друг другу и направлены вдоль $Y$ и $Z$. На рис. 7 приведена диаграмма зависимости реализации перемагничивания данной наноячейки с параметром $r_{0}=1$ от параметров действующего импульса. При близком расположении наночастиц диполь-дипольное взаимодействие выстраивает магнитные моменты вдоль оси наноячейки, т.е. вдоль оси $Z$, и действие импульса может привести либо к перемагничиванию обеих наночастиц, либо к сохранению конфигурации. На диаграмме затемненные области отвечают параметрам импульса, при которых реализуется перемагничивание всей наноячейки (для данных областей внизу диаграмма приведены начальная и конечная конфигурации наноячейки). Из диаграммы видно, что при длительности импульса $\tau_{0} \leq 2$ вблизи границы областей перемагничивания/неперемагничивания имеют место хаотические области, отвечающие параметрам, при которых результат действия импульса неопределен, т.е. под влиянием различных флуктуаций наноячейка может как перемагнититься, так и сохранить исходную конфигурацию. Данная особенность объясняется воздействием диполь-дипольного взаимодействия.

При ослаблении диполь-дипольного взаимодействия наряду с перемагничивание всей наноячейки реализуется ее частичное перемагничивание. На рис. 8 приведены аналогичные вышерассмотренным диаграммы для наноячеек с параметром $r_{0}=2(a, b)$ и $r_{0}=3(c, d)$. Черные и серые области диаграмм $(a, c)$ отвечают перемагничиванию либо только первой, либо только второй наночастицы. Исходные и соответствующие конечные конфигурации приведены вверху диаграмм. Черные области диаграмм $(b, d)$ отвечают перемагничиванию всей наноячейки (см. приведенные вверху конфигурации). Из

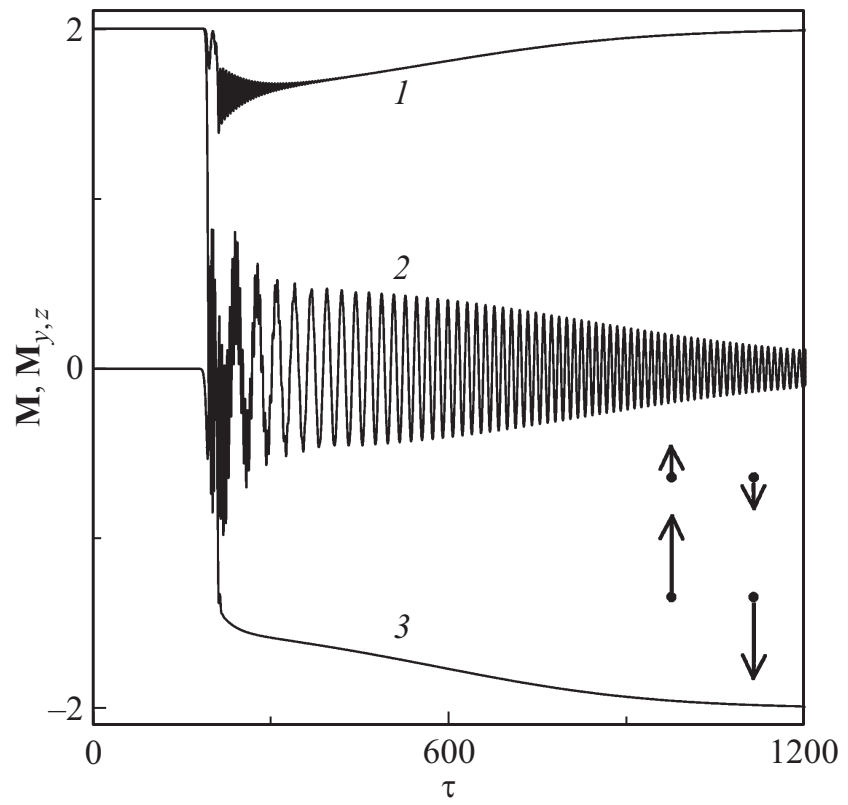

Рис. 6. Зависимости от времени модуля магнитного момента наноячейки (кривая 1) и его $y$-, z-компонент (кривые 2 и 3 ) для наночастиц с осями анизотропии вдоль оси $Z$ при действии импульса с $\tau_{0}=5, h_{0}=36 ; r_{0}=3$.

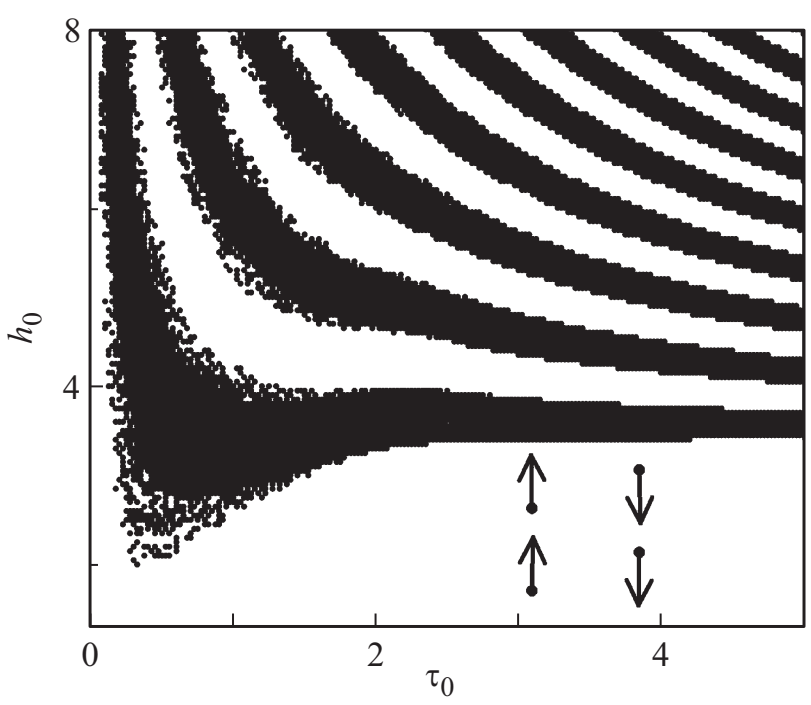

Рис. 7. Диаграмма перемагничивания наноячейки из двух одинаковых наночастиц с перпендикулярными осями анизотропии и параметром $r_{0}=1$; для отвечающих перемагничиванию затемненных областей приведены начальная и конечная конфигурации наноячейки.

рисунка видно, что при $r_{0}=2.0$ преобладают области бистабильности, т.е. области с неопределенным результатом действия импульса.

Области параметров импульса, однозначно отвечающие перемагничиванию только одной из наночастиц, появляясь при расхождении частиц $\left(r_{0}>1\right)$, по достижении некоторого значения $r_{0}$, начинают сужаться. При 


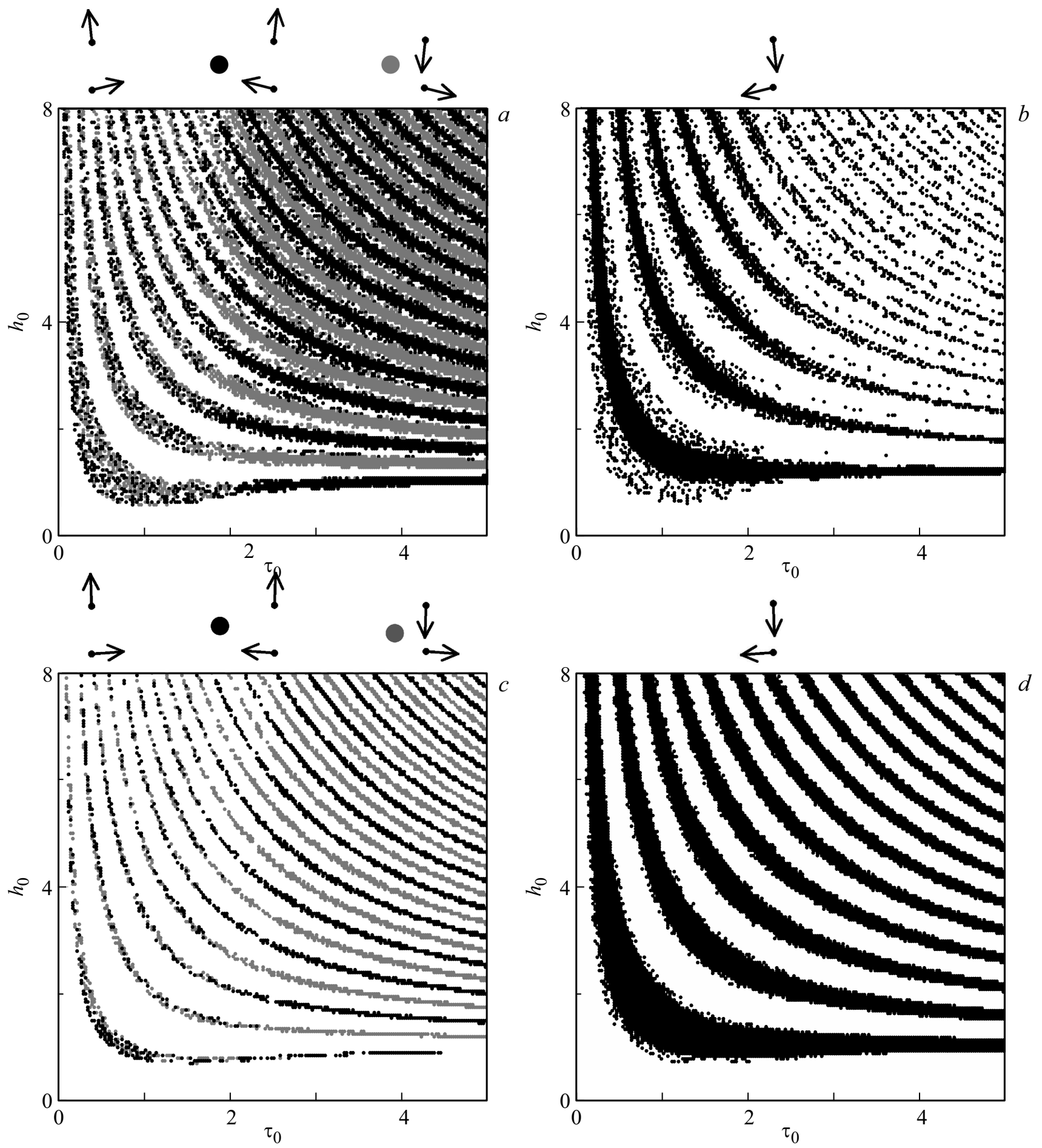

Рис. 8. Диаграммы перемагничивания наноячейки из одинаковых наночастиц с перпендикулярными легкими осями и параметром $r_{0}=2(a, b)$ и $r_{0}=3(c, d)$; черные и серые области диаграмм $(a, c)$ отвечают перемагничиванию только одной из наночастиц; диаграммы $(b, d)$ отвечают перемагничиванию всей наноячейки; исходные и соответствующие конечные конфигурации приведены вверху диаграмм.

этом они имеют место только в случае достаточно продолжительных импульсов $\left(\tau_{0} \geq 2\right)$. Области перемагничивания всей наноячейки наоборот: преобладают при коротких импульсах и расширяются при увеличении параметра $r_{0}$.
На рис. 9 для вышерассмотренной наноячейки с параметром $r_{0}=2$ представлены проекции на плоскость $Y Z$ траекторий магнитных моментов, входящих в нее наночастиц, при действии импульса с $h_{0}=1.0,2.5(a, d)$, $h_{0}=1.5(b, c), \quad \tau_{0}=3 \quad(a, c)$ и $\tau_{0}=1 \quad(b, d)$. В слу- 

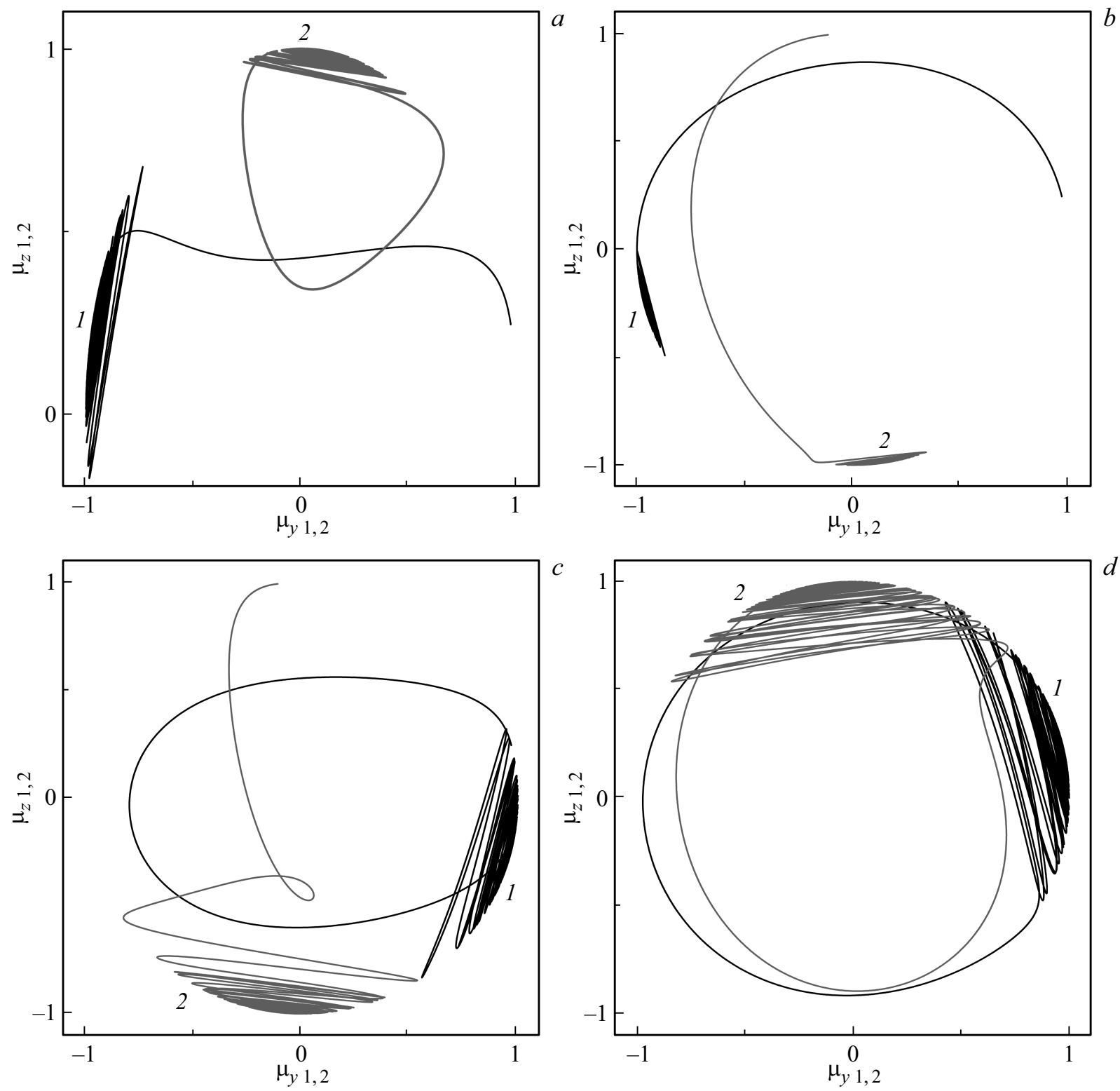

Рис. 9. Проекции на плоскость $Y Z$ траекторий магнитных моментов первой и второй наночастицы (кривые 1 и 2 ) при действии импульса с $h_{0}=1.0,2.5(a, d), h_{0}=1.5(b, c), \tau_{0}=3(a, c)$ и $\tau_{0}=1(b, d) ; \mu_{1,2}=1, r_{0}=2$.

чаях $(a)$ и $(c)$ реализуется перемагничивание только первой и только второй наночастицы, соответственно, в случае $(b)$ перемагничивается вся нанояччейка, и в случае $(d)$ после действия импульса наноячейка сохраняет исходную конфигурацию. Если параметры импульса отвечают центральным участкам областей диаграммы перемагничивания, прецессионная динамика отклика магнитных моментов является короткой $(b)$, и быстро устанавливается новое равновесное положение [21]. Приближение параметров к границе между областями диаграммы приводит у увеличению продолжительности и амплитуды прецессионной динамики отклика магнитных моментов на воздействие импульса $(d)$.

Для данных случаев импульсного воздействия на рис. 10 приведены зависимости от времени $y$-компонен- ты суммарного магнитного момента наноячейки. Видно, что амплитуда прецессионной динамики отклика магнитных моментов может отличаться в несколько раз при разных параметрах импульса (в зависимости от их расположения на диаграмме перемагничивания [22]). Наблюдаемые биения прецессии обусловлены влиянием диполь-дипольного взаимодействия между наночастицами ячейки.

Для осуществления количественных оценок приведем переход от безразмерных величин к размерным для наночастиц, состоящих из $N$ атомов железа: магнитный момент наночастицы $m \approx 2.2 \mu_{B} N$, где $\mu_{B}$ - магнетон Бора. Так, для устойчивой сферической конфигурации атомов железа $N=561$ и радиус наночастицы составляет $R=1.364 \cdot 10^{-7} \mathrm{~cm}$, 

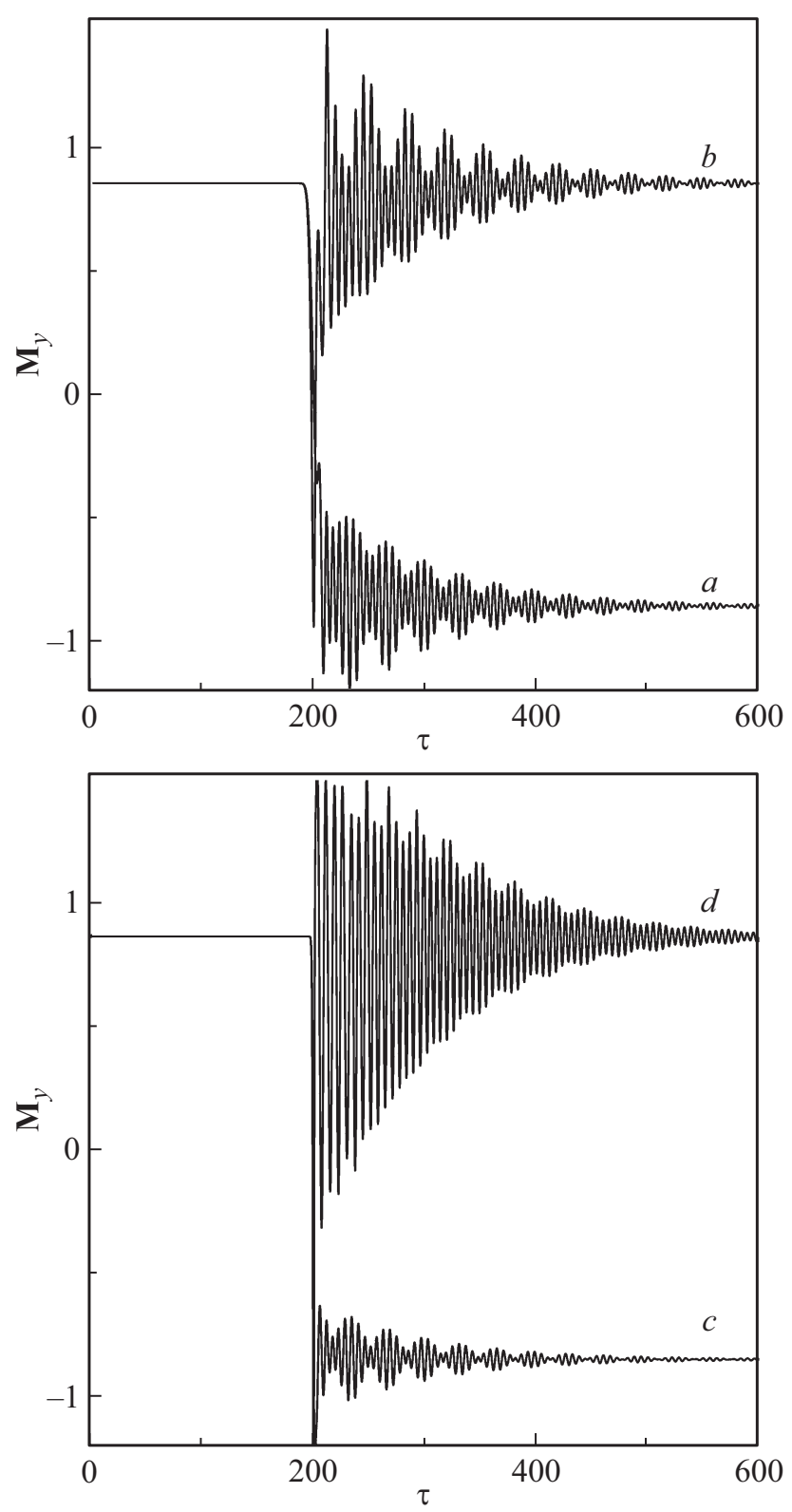

Рис. 10. Зависимости от времени $y$-компоненты суммарного магнитного момента наноячейки с $\mu_{1,2}=1, r_{0}=2$ и перпендикулярными осями анизотропии наночастиц при действии импульса с $h_{0}=1.0,2.5(a, d), h_{0}=1.5(b, c), \tau_{0}=3(a, c)$ и $\tau_{0}=1(b, d)$.

a $m \approx 1.145 \cdot 10^{-17} \mathrm{erg} / \mathrm{Oе}$ и $J \approx 1.08 \mathrm{kGs}$. С учетом $\gamma=1.76 \cdot 10^{7} \mathrm{Oe} \cdot \mathrm{s}^{-1}$ получаем следующие численные оценки для времени $t=\tau /(\gamma J) \approx 0.53 \tau$ ps, магнитного поля $H=J h \approx 1.08 h \mathrm{kOe}$, константы анизотропии $K_{i u}=J^{2} k_{i u} \approx 1.2 \cdot 10^{6} k_{i u} \mathrm{erg} / \mathrm{cm}^{3}$.

\section{5. Заключение}

Исследование ячейки, состоящей из двух наночастиц с одноосной магнитной анизотропией, показало, что отклик на импульсное воздействие и конечная конфигу- рация магнитных моментов ячейки существенно зависит от параметров импульса магнитного поля. При этом в случае одинаковых наночастиц с перпендикулярными осями анизотропии или в случае различных по величине магнитного момента наночастиц реализуется мультистабильное перемагничивание, когда в зависимости от длительности и амплитуды импульса устанавливается одна из четырех равновесных конфигураций наноячейки. При изменении параметров перемагничивающего импульса имеет место чередование условий реализации различных конечных конфигураций. В случае достаточно коротких импульсов возникают также области бистабильного перемагничивания, когда при одних и тех же параметрах под влиянием флуктуаций начальных условий могут устанавливаться различные конечные конфигурации магнитных моментов.

В случае перпендикулярных осей анизотропии наночастиц при коротких импульсах $\left(\tau_{0} \leq 2\right)$ реализуется перемагничивание всей наноячейки. Области параметров, отвечающие перемагничиванию только одной из наночастиц, преобладают при импульсах, достаточно большой продолжительности, и параметре ячейки $r_{0} \approx 2$. В случае параллельных осей анизотропии при большом диполь-дипольном взаимодействии (т.е. при малом параметре ячейки) перемагничивание обеих наночастиц преобладает в случае ориентации осей анизотропии вдоль оси наноячейки, а перемагничивание только одной из наночастиц - при перпендикулярной ориентации осей анизотропии оси наноячейки.

Продолжительность отклика наноячейки определяется расположением параметров импульса на диаграмме перемагничивания. Если параметры находятся вблизи центра характеристических области диаграммы, переходная динамика магнитных моментов непродолжительна и практически ограничивается временем действия импульса. Если же параметры импульса близки к границе между областями диаграммы, продолжительность прецессионной динамики отклика возрастает в несколько раз. При параметре $r_{0} \geq 4$ дипольдипольное взаимодействие становится слабым, и отклик магнитных моментов близок к случаю изолированных наночастиц. Таким образом, данная величина параметра ячейки задает требуемую постоянную дипольной решетки. Полученные результаты могут быть использованы при создании четырех уровневых элементов памяти на основе рассмотренных ячеек наночастиц.

\section{Конфликт интересов}

Авторы заявляют, что у них нет конфликта интересов.

\section{Список литературы}

[1] S.A. Gudoshnikov, B.Ya Liubimov, A.V. Popova, N.A. Usov. JMMM 324, 22, 3690 (2012).

[2] T. Kiseleva, S. Zholudev, A. Novakova, T. Grigoryeva. Comp. Struct. 138, 12 (2016). 
[3] M.F. Hansen, P.E. Jönsson, P. Nordblad, P. Svedlindh. J.Phys.: Condens. Matter 14, 19, 4901 (2002).

[4] A.M. Shuty̌̌, S.V. Eliseevay, D.I. Sementsov. Phys. Rev. B 91, 2, 024421 (2015).

[5] A.M. Shuty̌́, D.I. Sementsov. JMMM 401, 3, 1033 (2016).

[6] N. Eibagi, J.J. Kan, F.E. Spada, E.E. Fullerton. IEEE Magn. Lett. 3, 4500204 (2012).

[7] Е.3. Мейлихов, Р.М. Фарзетдинова. ФТТ 56, 12, 2326 (2014).

[8] H.W. Schumacher, C. Chappert, P. Crozat, R.C. Sousa, P.P. Freitas, J. Miltat, J. Fassbender, B. Hillebrands. Phys. Rev. Lett. 90, 1, 017201 (2003).

[9] H.W. Schumacher, C. Chappert, R.C. Sousa, P.P. Freitas, J. Miltat. Phys. Rev. Lett. 90, 1, 017204 (2003).

[10] A.V. Kimel, B.A. Ivanov, R.V. Pisarev, P.A. Usachev, A. Kirilyuk, Th. Rasing. Nature Phys. 5, 727 (2009).

[11] Satoh Takuya, Cho Sung-Jin, Iida Ryugo, Tsutomu Shimura, Kazuo Kuroda, Hiroaki Ueda, Yutaka Ueda, B.A.Ivanov, Franco Nori, Manfred Fiebig. Phys. Rev. Lett. 105, 7, 077402 (2010).

[12] А.Ю. Галкин, Б.А. Иванов. Письма в ЖЭТФ 88, 4, 286 (2008).

[13] Ю.И. Джежеря, В.П. Юрчук, К.О. Демишев, В.Н. Коренивский. ЖЭТФ 144, 6 (12), 1203 (2013).

[14] A. Sukhov, J. Berakdar. Phys. Rev. B 79, 13, 134433 (2009).

[15] В.В. Рандошкин, А.М. Салецкий, Н.Н. Усманов, Д.Б. Чопорняк. ФТТ 46, 3, 461 (2004).

[16] Е.И. Ильяшенко, О.С. Колотов, А.В. Матюнин, О.А. Миронец, В.А. Погожев. ЖТФ 76, 11, 140 (2006).

[17] Д.А. Балаев, А.А. Красиков, Д.А. Великанов, С.И. Попков, Н.В. Дубынин, С.В. Столяр, В.П. Ладыгина, Р.Н. Ярославцев. ФТТ 60, 10, 1931 (2018).

[18] Ю.И. Джежеря, К.О. Демишев, В.Н. Коренивский. ЖЭТФ 142, 2 (8), 318 (2012).

[19] А.М. Шутый, Д.И. Семенцов. Физика металлов и металловедение 121, 1, 1 (2019).

[20] А.М. Шутый, Д.И. Семенцов. Письма в ЖЭТФ 108, 11, 761 (2018).

[21] А.М. Шутый, Д.И. Семенцов. ФТТ 61, 10, 1783 (2019).

[22] A.M. Shuty, S.V. Eliseeva, D.I. Sementsov. Superlattic. Microstruct. 132, 106158 (2019).

[23] А.Г. Гуревич, Г.А. Мелков. Магнитные колебания и волны. Наука, М. (1994).

Редактор Т.Н. Василевская 